\title{
La identificación de las emociones, corporalidad y lenguaje como una herramienta para el aprendizaje en estudiantes de educación superior
}

\section{The identification of emotions, corporeality and language as a tool for learning in higher education students}

\author{
DOI: $10.46932 / s f j d v 2 n 4-010$
}

Received in: Jun1st, 2021

Accepted in: Sep 30th, 2021

\author{
Carlos Alfredo Damián García \\ Dr. En Investigación Educativa \\ Esc. Normal Experimental \\ "Salvador Varela Reséndiz" \\ Av. de la Normal S/N \\ Juchipila, Zac. México \\ E-mail: cabritoreposado@gmail.com
}

\section{RESUMEN}

El presente trabajo de investigación está enfocado en identificar las emociones,corporalidad y lenguaje presentes en el proceso de aprendizaje de estudiantes de nivel superior. El estudio se realizó a 24 alumnos de la licenciatura en Educación primaria de la Escuela Normal Experimental "Salvador Varela Resendiz" de Juchipila Zacatecas. Es un estudio cuantitativo no experimental transeccional descriptivo, donde se aplicó el test de perfiles de personalidad DISC, el test de estilos de aprendizaje de McCarthy y un test de coeficiente emocional basado en David Goleman. Se identificó y describió los perfiles de personalidad, estilos de aprendizaje y coeficiente emocional que más predominan en el grupo de alumnos, encontrándose que, en la mayoría de los casos, un tipo de personalidad se asocia con un estilo de aprendizaje y con ciertas habilidades emocionales, creando una relación significativa.

Palabras clave: Coeficiente emocional, estilos de aprendizaje, personalidad.

\begin{abstract}
This research work is focused on identifying the emotions, corporeality and language present in the learning process of higher level students. The study was carried out on 24 students of the degree in Primary Education of the "Salvador Varela Resendiz" Experimental Normal School of Juchipila Zacatecas. It is a descriptive non-experimental transectional quantitative study, where the DISC personality profiles test, the McCarthy learning styles test and an emotional coefficient test based on David Goleman were applied. The personality profiles, learning styles and emotional coefficient that most predominate in the group of students were identified and described, finding that, in most cases, a personality type is associated with a learning style and with certain emotional abilities , creating a meaningful relationship.
\end{abstract}

Keywords: Emotional coefficient, learning styles, personality.

\section{INTRODUCCIÓN}

La Escuela Normal Experimental “Salvador Varela Reséndiz” (ENESVR) de Juchipila Zacatecas se fundó en 1977. Se ubica en un contexto urbano, concentrando a 212 alumnosde la región sur del estado. 
La visión de dicha institución es mejorar la calidad de la educación, impactando en la formación de licenciados en educación primaria y educación prescolar. Nos interesó realizarla investigación en dicha institución, ya que contempla en su malla curricular del plan de estudios 2018, los cursos de "Educación Socioemocional" y "Estrategias para el desarrollosocioemocional", siendo programas innovadores en el subsistema de educación normal; porlo tanto, no se cuenta con diagnósticos institucionales que reporten información de educación en las emociones.

En base a dichos antecedentes, el objetivo de este estudio fue identificar las emociones,corporalidad y lenguaje como una herramienta para el aprendizaje en estudiantes de educación superior.

En una investigación realizada en 2018, en la Universidad de Buenos Aires, Argentina "Eltrabajo sobre las emociones en la formación de educadores en el nivel superior", permitieron identificar 4 modalidades: los juegos proyectivos, la retroalimentación de elucidación, la retroalimentación descriptiva y la retroalimentación propositiva. Dicha investigación encontró que las estrategias anteriores influyeron en emociones positivas en el alumnado [1].

\section{MATERIALES Y MÉTODO}

El presente es una investigación no experimental transeccional descriptiva porque recolecta datos en un solo momento, en un tiempo único (Liu, 2008 y Tucker, 2004). Dondese describen y analizan las variables personalidad, coeficiente emocional y el estilo de aprendizaje, para observar su incidencia e interrelación en un momento dado [2].

Participó una población de 24 alumnos del segundo semestre de la Licenciatura de educación primaria de la ENESVR.

Se midió la personalidad a través del test de perfiles de personalidad DISC-UPF, evaluando cuatro factores de conducta: Dominance (dominancia), Influence (influencia social), Steadiness (estabilidad, paciencia, perseverancia, cooperación) y Compliance (estructura, obediencia, precisión) [3].

Otro test nos permitió evaluar el coeficiente emocional por su conducta, calibrado por elmaestro Quirino García Guillen del Tecnológico de Monterrey con más de 10 años de aplicación, basado en la teoría de la inteligencia emocional de Daniel Goleman, donde propone que el coeficiente emocional está compuesto de autodominio, autoconocimiento, conciencia emocional y empatía [4].

El último test aplicado fue un inventario de estilos de aprendizaje de McCarthy 1987, donde se detectó preferencias referentes a los estilos: observando, conceptualizando, haciendo y experimentando [5]. 


\section{RESULTADOS}

Fig. 1. Perfiles de personalidade

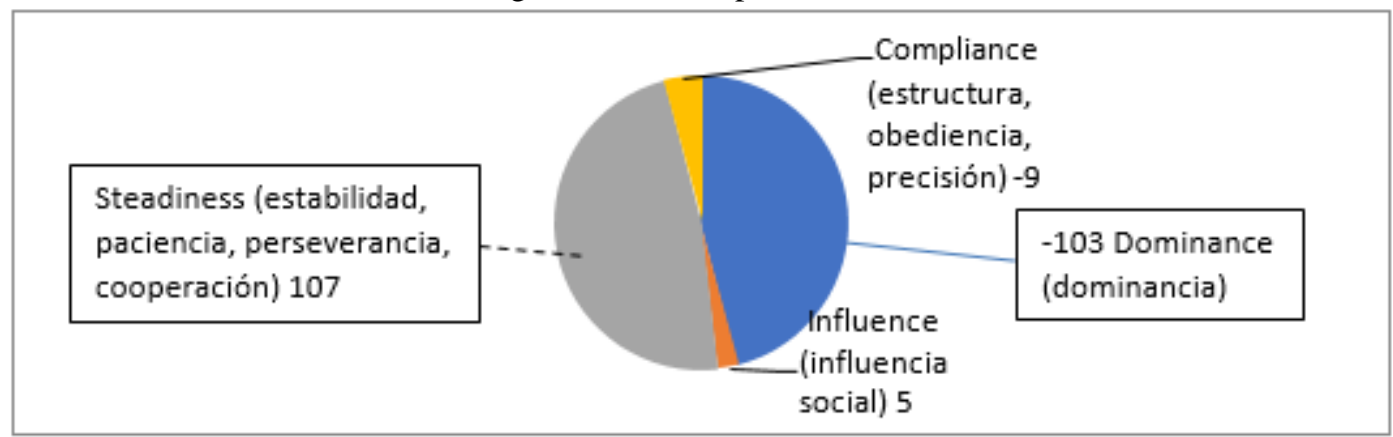

El test de perfiles de personalidad DISC-UPF permitió que los estudiantes se conozcanmejor y nosotros al estudiante. Como se puede observar, el grupo de 24 estudiantes tiene una predominancia en el factor Steadiness con 107 y se encontró que en dichos estudiantesla actitud que menos presentan es el factor Dominance con -103 .

Tabla 1. Perfiles de personalidad

\begin{tabular}{|c|c|c|c|c|}
\hline & Influencia & Dominancia & Estabilidad & Cumplimiento \\
\hline Válido & 24 & 24 & 24 & 24 \\
\hline Perdidos & 0 & 0 & 0 & 0 \\
\hline Media & .21 & -4.29 & 4.46 & -.38 \\
\hline Mediana & 1.00 & -3.50 & 5.50 & -1.50 \\
\hline Moda & -4 & $-8^{2}$ & 7 & -5 \\
\hline
\end{tabular}

Como se puede apreciar, al calcular el promedio de cada factor de la conducta se encontró que el factor Steadiness se encuentra al alta con un valor de 4.46 y a la baja conel factor Dominance con un valor de -4.29 .

De igual manera con el test "inventario de estilos de aprendizaje" nos permitió detectar las preferencias de los estudiantes, siendo las siguientes: 
Fig. 2. Estilos de aprendizaje

\begin{tabular}{|cccc|}
\hline & & 936 & 934 \\
\hline 843 & 889 & & \\
\hline & & & \\
& & & \\
\hline & & & \\
\hline
\end{tabular}

Como se puede observar en los test de los 24 alumnos, el estilo de aprendizaje que máspredomina es el de conceptualizando-haciendo (triángulo) con 936 y el que se encuentra ala baja es el de experimentando-haciendo (estrella) con 843.

Tabla 2. Estilos de aprendizaje

\begin{tabular}{|c|c|c|c|c|}
\hline & Estrella & Círculo & Triángulo & Cuadrado \\
\hline Válido & 24 & 24 & 24 & 24 \\
\hline Perdidos & 0 & 0 & 0 & 0 \\
\hline Media & 35.13 & 37.04 & 39.00 & 38.92 \\
\hline Mediana & 36.00 & 37.00 & 39.00 & 38.50 \\
\hline Moda & 36 & 31 & 41 & 40 \\
\hline
\end{tabular}

Al calcular el promedio de la puntuación se encontró que el estilo de aprendizaje conceptualizando-haciendo (triángulo) está al alta con un promedio de 39 y el factor experimentandohaciendo (estrella) se ubicó a la baja con un 35.13.

Por último, el test "Coeficiente emocional por su conducta" permitió conocer la dominancia que se tiene en autodominio, autoconocimiento, conciencia emocional, empatía y coeficiente emocional elevado.

Fig. 3. Coeficiente emocional

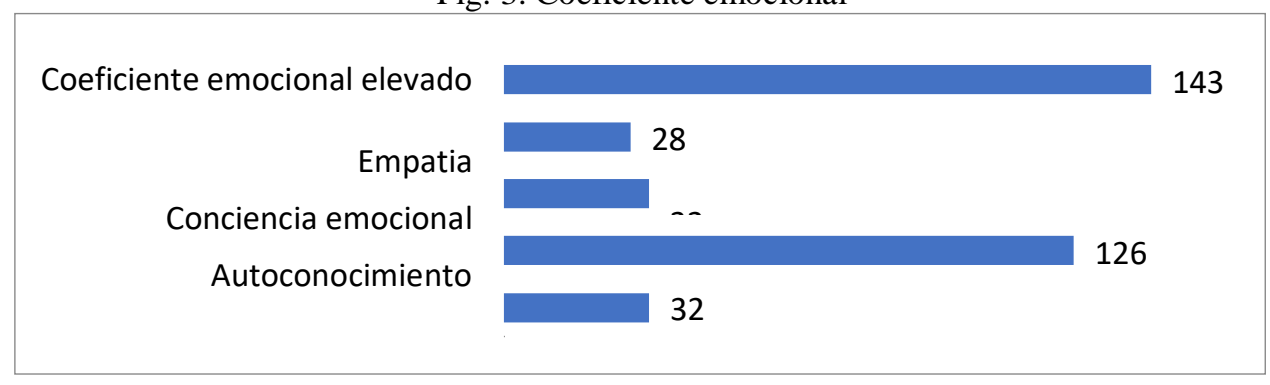

Entre estas 5 habilidades, predomina el coeficiente emocional elevado con un totalde 143 y el 
que se encuentra a la baja es la empatía con 28.

Tabla 3. Coeficiente Emocional

\begin{tabular}{|c|c|c|c|c|c|}
\hline & $\begin{array}{c}\text { Auto } \\
\text { dominio }\end{array}$ & $\begin{array}{c}\text { Auto } \\
\text { conocimiento }\end{array}$ & $\begin{array}{l}\text { Conciencia } \\
\text { Emocional }\end{array}$ & Empatía & $\begin{array}{l}\text { Coeficiente E. } \\
\text { Elevado }\end{array}$ \\
\hline $\begin{array}{ll}\mathrm{N} & \text { Válido }\end{array}$ & 24 & 24 & 24 & 24 & 24 \\
\hline Perdidos & 0 & 0 & 0 & 0 & 0 \\
\hline Media & 1.33 & 5.25 & 1.33 & 1.17 & 5.96 \\
\hline Mediana & 1.00 & 5.00 & 1.00 & 1.00 & 6.00 \\
\hline Moda & 1 & $4^{\mathrm{a}}$ & 1 & & $4^{\mathrm{a}}$ \\
\hline
\end{tabular}

Calculando el promedio se encontró que, el factor dominante fue el coeficiente emocional elevado con 5.96 y a la baja se identificó el factor de empatía con 1.17

\section{DISCUSIÓN (O ANÁLISIS DE RESULTADOS)}

Como se puede observar en la Fig.1. Perfiles de personalidad, el 60\% de los alumnosson pacientes, compasivos y leales con los que les rodean. Están interesados por los problemas de los demás y son buenos para cubrir roles de apoyo. Tienen un enfoque perseverante con facultades de concentración. Se resisten a los cambios y trabajan mejor cuando se les da instrucciones claras y mucho apoyo. Toman el rol de pacificadores, conciliadores.

En contraste, se encontró que en dichos estudiantes el $40 \%$ tienen control, poder y asertividad. Es el factor de la proactividad y el control. Es una mezcla de aspectos positivosy negativos. De los positivos encontramos independencia, motivación para ser exitosos enlograr lo que quieren. Sin embargo, también pueden ser de temperamento fuerte y agresivos. A estos alumnos les gusta tener autoridad y poder sobre los demás, son competitivos, ambiciosos y le gustan los desafíos. No confían fácilmente en losdemás y tratarán de tener éxito por sus propios medios, sin ayuda. En caso de necesitarla,darán ordenes en vez de pedir colaboración.

De igual manera en la fig. 2. Estilos de aprendizaje, donde predomina el perfil de las personas que aprenden conceptualizando-haciendo, son excelentes para la planeación, reflexión y crítica; su pensamiento es lógico, tienen habilidad para el análisis, son eficientes, organizados, estudiosos y precisos. Les gusta aprender. Sus debilidades son larigidez y la dificultad que tienen para llevar a la práctica sus ideas. Habitualmente tienen problemas con personas desorganizadas.

Por otro lado, se ubicó a la baja el perfil de las personas que aprenden experimentando-haciendo, su fortaleza es la capacidad de innovar. Son creativos, intuitivos, les gusta explorar nuevas posibilidades y la aventura. Sus debilidades son el carácter impulsivo y la poca organización, normalmente tienen problemas con personas rígidas.

Por ultimo en la Fig. 3. Coeficiente emocional, se encuentra al alta el coeficiente emocional 
elevado (que son las respuestas de CE esperado) y a la baja la empatía, en la cual se debe trabajar más.

Como lo menciona (Suárez-Carreño, 2021) la identificación de las emociones es una tarea compleja, puesto que observaron cambios de hasta 3 emociones en un periodo corto de 10 segundos, considerando que las emociones no son muy constantes en los sujetos. Sin embargo, se pueden aplicar ciertos instrumentos que nos pueden arrojar información más específica, que nos permita interpretar con mayor proximidad al lenguaje corporal como el espectro de voz, la fisionomía del rostro, el análisis de video, así como una variedad de test o inventarios [6].

\section{CONCLUSIONES Y RECOMENDACIONES}

Se identificó que en el grupo predomina la personalidad de estabilidad, perseverancia y cooperación, tienden a estar interesados por los problemas de los demás y son buenos para cubrir roles de apoyo. También predomina el estilo de aprendizaje conceptualizando-haciendo, lo que significa que son excelentes para la planeación, la reflexión y la crítica; su pensamiento es lógico, tienen habilidad para el análisis, son eficientes, organizados, estudiosos y precisos.Les gusta aprender. Así mismo, predomina el coeficiente emocional elevado, que es el ideal.

También se pudo observar que los alumnos con personalidad Dominance, se refiere a gente que le gusta tener autoridad y poder sobre los demás, son competitivos, ambiciosos y les gustan los desafíos. No confían fácilmente en los demás y tratarán de teneréxito por sus propios medios, sin ayuda. Su estilo de aprendizaje es experimentando- haciendo, son de carácter impulsivo y poca organización, normalmente tienen problemas con personas rígidas y en su coeficiente emocional les hace falta trabajar duro, para poder ser empáticos.

Por lo tanto, se observó que, en la mayoría de los casos, un tipo de personalidad se asocia con un estilo de aprendizaje y con ciertas habilidades emocionales, creando una relación significativa.

\section{RECONOCIMIENTOS (O AGRADECIMIENTOS)}

Expreso mi agradecimiento al Tecnológico De Monterrey campus Querétaro por la facilitación de los test y a la Universidad Autónoma de Zacatecas campus Jalpa por las facilidades y el apoyo otorgado para la realización de la estancia de verano. 


\section{REFERENCIAS}

[1] L. Sánchez, Troussel, \& M. Manrique, (2018). El trabajo sobre las emociones en la formación de educadores en el nivel superior. REXE. Revista de Estudios y Experiencias en Educación, 17 (34), 139147. http://dx.doi.org/https://doi.org/10.21703/rexe.20181734lsanchez2

[2] R. Hernández. Metodología de la investigación, Ed. McGraw-Hill. México D.F., 2014.

[3] H. Salinas. (S/F). "Perfiles de la PERSONALIDAD Reseñas generales del DISC". Universidadpara la familia (UPF). http://universidadparalafamilia.org/Materiales/Resena_DISC-UPF.pdf

[4] D. Goleman. Inteligencia emocional: Kairos 2010. http://www.codajic.org/sites/www.codajic.org/files/Inteligencia\%20Emocional\%20\%20Daniel\%2 0Goleman.pdf

[5] B. McCarthy, (1987). 4MAT Systema (sistema de formato). http://www.cca.org.mx/profesores/cursos/cep21/modulo_2/modelo_4mat.htm

[6] F. Suárez-Carreño (2021). Soportes neurales de las emociones: un acercamiento descriptivo. Vol. 2 No. 4(2021): South Florida Journal of Development, Miami, p.5560-5567, v. 2, n. 4, jul./sep. 2021ISSN 2675-5459 https://southfloridapublishing.com/ojs/index.php/jdev/article/view/714/647 